ORIGINAL ARTICLES

\title{
Thoracic epidural analgesia vs. intravenous analgesia after lung transplant
}

\author{
Enten $\mathrm{G}^{1}$, Puri $\mathrm{S}^{2}$, Patel $\mathrm{K}^{3}$, Stachura $\mathrm{Z}^{3}$, Schwaiger $\mathrm{E}^{3}$, Patel $\mathrm{P}^{3}$, Melloni $\mathrm{G}^{4}$, Camporesi $\mathrm{EM}^{* 1,2}$ \\ ${ }^{1}$ TEAMHealth Research Institute, Tampa, FL, United States \\ ${ }^{2}$ TEAMHealth Anesthesia, Tampa, FL, United States \\ ${ }^{3}$ University of South Florida Department of Pulmonology, Tampa, FL, United States \\ ${ }^{4}$ Havard School of Public Health, Boston, MA, United States
}

Received: July 8, 2019

DOI: $10.5430 /$ css.v5n2p4
Accepted: August 6, 2019

URL: https://doi.org/10.5430/css.v5n2p4
Online Published: August 20, 2019

\begin{abstract}
Objective: Few reports have evaluated postoperative continuous thoracic epidural analgesia on patients who received a lung transplant. This analgesic modality may facilitate extubation, early ambulation, and achieve adequate pain control with minimization of opioid use. An opioid sparing technique could minimize the side effects of opioids such as ileus, constipation, and somnolence.

Methods: A retrospective chart review following local IRB approval was performed. A total of 97 patients' charts were collected, from April 2015 to March 2017. Forty-eight patients received T6-7 epidural, and forty-nine patients received standard intravenous (IV) analgesia. Outcome measures collected included length of intensive care unit stay, total duration of hospitalization, need for reintubation or noninvasive intermittent positive pressure ventilation (NIPPV), need for IV lidocaine gtt, and total narcotics consumption during hospitalization in milligrams of morphine equivalents (MME).

Results: Both groups were comparable in age, BMI, and race/gender distribution. Additionally, patient pain requirements were comparable between groups. However, a significantly smaller proportion of thoracic epidural patients required NIPPV post-operatively, (20.4\%, 53.2\%: $p=.0015)$. Further, the number of patients requiring reintubation was almost halved, $(12.5 \%$, 21.3\%: NS). Patients receiving thoracic epidural also experienced shorter ICU times $(p=.0335)$ and on average, an overall reduced length of stay by six days.

Conclusions: For patients undergoing lung transplant, epidural analgesia is a viable alternative to IV pain control. Further, it significantly reduced respiratory depression and length of stay in the ICU. More refined comparisons can be made by conducting a precise prospective study with a more structured protocol in place.
\end{abstract}

Key Words: Lung transplant postoperative analgesia, Thoracic epidural postoperative analgesia, Intravenous opioids

\section{INTRODUCTION}

Lung transplantation is becoming an increasingly common intervention for those who are suffering from end-stage lung disease. Over the past decades, worldwide lung transplantation numbers have dramatically increased from a meager
45 transplants in 1987 to greater than 3,700 transplants in 2011. ${ }^{[1]}$ While transplant numbers have increased exponentially over the years, one year and three year survival rates within the United States have remained relatively consistent over the past decade, $85 \%$ and $65 \%$ respectively. ${ }^{[1]}$ In the

\footnotetext{
*Correspondence: Camporesi EM; Email: ecampore@ @ealth.usf.edu; Address: TEAMHealth Anesthesia, 1 Tampa General Circle, Suite A327, Tampa, Florida, 33606, United States.
} 
field of thoracic surgery, quality of pain management has been directly correlated with patient outcomes. ${ }^{[2,3]}$ Acute perioperative pain may trigger the surgical stress response causing postoperative myocardial ischemia and new arrhythmias, increased peripheral vascular resistance, and reduced splanchnic circulation thereby extending time spent in the intensive care unit (ICU). ${ }^{[4]}$ Post lung transplant, undertreated perioperative pain has also been linked to poor respiratory and accessory muscle strength. ${ }^{[5,6]}$ Poor respiratory effort often requires prolonged intubation and mechanical ventilation, which is associated with the development of pneumonia and atelectasis. ${ }^{[4,5]}$ Ultimately this leads to a protracted recovery and a longer hospital length of stay.

Further, development of new-onset chronic pain is also common in those undergoing thoracic surgery; a study by Feltracco et al., shows more than half of all thoracotomy patients who reported acute postsurgical pain developed chronic pain at the surgical site. ${ }^{[7]}$ Chronic pain post thoracic surgery has been associated with significantly decreased physical function and worse quality of life. ${ }^{[8]}$ Mitigating acute perioperative pain in thoracic surgery plays a key role in bettering patient outcomes, enhancing recovery, and reducing hospital costs.

Thoracic epidural analgesia (TEA) is now regarded as a critical component of multimodal pain control for lung transplantation. However, the clinical benefit of TEA on patients undergoing lung transplantation and the optimal timing of epidural placement remains unclear. This study evaluates the safety and efficacy of post-operative placement of T6-T7 thoracic epidural for lung transplant and compares this modality to intravenous (IV) analgesia.

\section{MethodS}

A retrospective chart review following IRB approval from the University of South Florida was performed (Pro00037269). Data were evaluated on the effects of continuous epidural analgesia initiated postoperatively as compared to standard IV analgesia. All patients receiving post-operative thoracic epidural analgesia were recruited upon request of surgeons at Tampa General Hospital from April 2015 to March 2017. Inclusion criteria were age over 18 , and received single or bilateral lung transplantation. Forty-eight patients received T6-T7 epidural, and forty-nine patients that had contraindications for placement of thoracic epidural, such as coagulopathy or anticoagulation, were given IV analgesia. Primary outcomes were length of time spent in the ICU, total duration of hospitalization, need for reintubation or noninvasive intermittent positive pressure ventilation (NIPPV), need for IV lidocaine $\mathrm{gtt}$, and total narcotics consumption during hospitalization in milligrams of morphine equivalents (MME).

Published by Sciedu Press
Placement of epidural catheters followed the standard of the American Society of Regional Anesthesia guidelines. After verifying coagulation studies were acceptable (PT/INR $>1.5$, Platelets $>100,000$ ), patient received epidural 6-8 hours after ICU admission while intubated and sedated on propofol drip. Thoracic Epidural was inserted at T6-T7 until loss of resistance was observed. Catheter position was verified with a test infusion of lidocaine +3-4 ccs of epinephrine seeking no change in heart rate after bolus injection. The initial drip rate in this epidural space consisted of ropivacaine 0.1 $\mathrm{mg} / \mathrm{cc} 8-9 \mathrm{ml} / \mathrm{hr}$ with a $2.5 \mathrm{cc}$ bolus q $30 \mathrm{~min}$ on request for persistent pain. Maximum infusion rate was 13-14 ml/hour. Ropivacaine was increased to $0.2 \mathrm{mg} / \mathrm{cc}$ if patient reported a pain score of 6 or more on a $10 \mathrm{~cm}$ visual analogue scale or if an increase in blood pressure was observed.

Postoperative pain course for the IV analgesia group utilized hydromorphone PCA in standard dosage and lockout regimens. Transdermal fentanyl, oral tramadol and Norco (5/325 hydrocodone/acetaminophen) were given on an as-needed basis.

Continuous data are presented in the form of mean \pm standard deviation $(S D)$. Categorical data are presented as proportions. Continuous data were analyzed using a two tailed $t$-test with a significance of $p<.05$. Categorical data were analyzed using a Fisher's exact test with a significance of $p$ $<.05$. Data of further clinical interest were analyzed for their effect size using Cohen's d or Cramer's v for continuous data and categorical data respectively.

\section{Results}

Postoperative management of ventilator support was the same for both groups of patients and was equally coordinated and supervised by pulmonary critical care services (PK) with pain management supervised by anesthesia and pain services (PS). Both groups were comparable in age, BMI and race/gender distribution (see Table 1). When compared to the patient series receiving IV pain management, patients receiving thoracic epidural did not show a significant reduction in total narcotics requirement or a significant reduction in the number of patients requiring lidocaine gtt (see Table 1). However, respiratory improvements were seen, demonstrated by a significantly smaller proportion of patients requiring non-invasive positive pulmonary ventilation (NIPPV) post-operatively, $20.4 \%$ as compared to $53.2 \%$ ( $p=$ .0015). Further, the number of patients requiring reintubation was almost halved, 6 (48) vs. 10 (49) respectively. While not statistically significant, this may be of clinical interest in future studies considering its effect size ( $\mathrm{v}>0.1$, OR 1.79). Patients receiving thoracic epidural also experienced shorter ICU times ( $p=.0335)$ and on average, an overall reduced length of stay by six days $(d>0.3)$. 
Table 1. Statistical comparison of patient characteristics after lung transplant

\begin{tabular}{|c|c|c|c|}
\hline & $\begin{array}{l}\text { Intravenous Analgesia } \\
(n=49)\end{array}$ & $\begin{array}{l}\text { Epidural Analgesia } \\
(n=48)\end{array}$ & $p$-value \\
\hline Age & $57.8(12.9)$ & $57.4(14.7)$ & NS \\
\hline Gender Distribution (\% Male) & $71 \%$ & $54 \%$ & NS \\
\hline Body Mass Index & $25.5(4.7)$ & $26.1(4.0)$ & NS \\
\hline Race Distribution (\% White) & $93.9 \%$ & $97.9 \%$ & NS \\
\hline Percent Receiving Double Lung Transplant & $73.5 \%$ & $70.8 \%$ & NS \\
\hline Surgery Duration (min) & $464.1(124.9)$ & $407.0(121.9)$ & $.0257^{*}$ \\
\hline Time spent in ICU (days) & $13.6(16.3)$ & $7.9(9.6)$ & $.0335^{*}$ \\
\hline Length of hospital stay (days) & $23.7(20.3)$ & $17.7(13.1)$ & .0874 \\
\hline Percent of Patients Needing Reintubation & $21.30 \%$ & $12.50 \%$ & .2733 \\
\hline Percent of Patients Needing (NIPPV) & $53.20 \%$ & $20.80 \%$ & $.0015^{* *}$ \\
\hline Percent of Patients Receiving Lidocaine gtt & $14.90 \%$ & $8.30 \%$ & .3364 \\
\hline Post-operative Narcotics Consumption (MME) & $400.4(668.6)$ & $549.6(471.0)$ & .2099 \\
\hline
\end{tabular}

Note. Data presented as Mean $\pm(S D)$ or as a percentage of individual sample size; ${ }^{*}$ indicates $p<.05 ;{ }^{* *}$ indicates $p<.005 ;$ NS - Not Significant

\section{Discussion}

Inadequate pain relief post-surgery has been associated with increased post-operative complications. Available analgesics that have been used to address the patient's need for pain control include: IV opioids, nonsteroidal antiinflammatory agents (NSAIDs), and acetaminophen. Often hypovolemic, patients post lung transplant make poor candidates for NSAID use. As cyclooxygenase (COX) inhibitors, NSAIDs present an increased risk for acute kidney injury. ${ }^{[9]}$ They additionally may increase risk of surgical site infection by impairing thromboxane-dependent platelet aggregation potentially resulting in prolonged sternal wound healing in double lung transplantation. ${ }^{[10,11]}$ Alternatively, these issues may be sidestepped through the administration of acetaminophen; however sufficient analgesia is typically not achieved through this alone. As such, opioids are typically a staple of postoperative pain management protocols With the dosages required to maintain satisfactory analgesia, opioid induced side effects such as postoperative nausea and vomiting, urinary retention, constipation, and respiratory depression can become a concern. Additionally, prolonged use of opioid analgesics may lead to chronic pain, opioid tolerance, and dependence.

Neuraxial anesthesia provides an alternative to the above mentioned methods. However there is currently a growing debate in the literature regarding the optimal mode of delivery of local anesthetic. ${ }^{[12,13]}$ While TEA is currently the gold standard in perioperative pain management for thoracic surgery, paravertebral blocks have been shown to be an effective method of pain control post-thoracotomy and lung transplantation. ${ }^{[14]}$ Whether paravertebral blocks pro- vide equivalent analgesia to TEA is under debate. A recent Cochrane review suggests that paravertebral block was equivalent in terms of pain management to thoracic epidural and had fewer minor complications. ${ }^{[15]}$ However, this has yet to be proven with a prospective randomized controlled trial. Conversely, Teeter et al., argues the literature supporting equivalent analgesia between both modalities often consists of poorly constructed studies with suboptimal thoracic epidural concentrations. Furthermore, Teeter et al., has argued that thoracic epidural may lead to less chronic pain. ${ }^{[12]}$

For now, TEA remains the gold standard for multi-modal pain management during lung transplantation. A 2005 metaanalysis by $\mathrm{Wu}$ et al showed for the first 72 hours after surgery TEA produced significantly lower pain scores and reduced narcotics requirements when compared to IV patientcontrolled analgesia (PCA) in patients with traumatic thoracic injury with rib fracture. ${ }^{[16]}$ Similarly, Sztain et al. demonstrated a reduction in opioid requirements after videoassisted thoracoscopic lobectomy. Compared to patients receiving liposomal bupivacaine, patients receiving T6-7 epidural for lobectomy on average required $43.3 \%$ less opioids within the first $48 \mathrm{hrs}$ of their surgery. ${ }^{[17]}$ More specifically, TEA has been shown to improve outcomes in patients after lung transplantation. ${ }^{[2,4,7]}$ Patients receiving TEA have shown reduced duration and need of mechanical ventilation, shortened length of ICU stay, and decreased number of respiratory complications. ${ }^{[18]}$ Patients receiving epidural in this study show improved respiratory outcomes as well, showing a significantly lower need for NIPPV and a lower frequency of reintubation. While the need for reintubation was not significantly lower in the TEA cohort, the frequency of its 
occurrence was almost halved; significance in this case is obscured by sample size as the need for reintubation occurs in only a small subset of our sample population.

Post major thoracic trauma and surgery, TEA has been demonstrated to improve patient satisfaction, ${ }^{[19]}$ reduce narcotics consumption, and reduce the development of chronic pain as compared to IV PCA. ${ }^{[20]}$ While our results do not support reduced narcotic consumption, the results are confounded by several heavy users dramatically increasing the variance of the sample. This could be a result of these patients having developed opioid tolerance prior to transplantation. A prospective study performed by Michel-Cherqu et al. characterizing the pain of 143 patients recently placed on the waiting list for lung transplantation showed that $59 \%$ were experiencing chronic pain prior to their surgery. Of those 85 patients, $14 \%$ were using some form of opiate to and $2 \%$ were using daily opioids to manage their pain. ${ }^{[21]}$ However, as we are unable to confirm prescription opioid usage prior to surgery we cannot rightfully exclude these patients from analysis. Additionally due to the nature of retrospective chart review, data concerning patient satisfaction and pain was either inconsistent, inaccurate, or incomplete for many patients reviewed, as such its collection and analysis was precluded from this study. This may be a contributing confound to our narcotics consumption data. While SOP dictates that patients reporting a VAS pain score of 6 or higher be given supplementary analgesia and pain management was handled by the same nursing staff in both groups, we cannot quantitatively confirm equivalent analgesia between groups.

The benefits of TEA do not however come without certain constraints. Patients undergoing lung transplantation on cardiopulmonary bypass are heparinized, as such the risk of epidural hematoma post catheter placement must be taken into account. A review of 4,583 epidural catheterizations in patients heparinized for cardiopulmonary bypass performed by Ho et al. estimates the risk of hematoma formation postcatheterization at a minimum of $1: 150,000$ catheterizations and maximum of 1:1,500 catheterizations. ${ }^{[22]}$ While there are several confirmed cases of spontaneous hematoma formation associated with neuraxial anesthesia in procedures utilizing cardiopulmonary bypass, ${ }^{[23]}$ to date, paraplegia, major neurological damage, and/or death due to perimedullary bleeding post-catheterization for lung transplantation have yet to be reported. ${ }^{[7]}$ In current practice, we have removed TEA from our protocols as we have started to utilize cardiopulmonary bypass for lung transplant cases. In the future, given the pulmonary and pain benefits that have been associated with TEA, we may reintroduce this analgesic method into our practice.

\section{Conclusions}

Our study indicates that for patients undergoing bilateral or single lung transplant, thoracic epidural anesthesia is a viable alternative to IV patient controlled analgesia for postoperative pain management. Further, it significantly reduced respiratory depression and length of stay in the ICU. More refined comparisons can be made by conducting a precise prospective study with a more structured protocol in place.

\section{CONFLicts OF INTEREST Disclosure}

The authors declare they have no conflicts of interest.

\section{REFERENCES}

[1] Klesney-tait J, Eberlein M, Geist L, et al. Starting a lung transplant program: a roadmap for long-term excellence. Chest. 2015; 147(5): 1435-1443. PMid: 25940255. https://doi.org/10.1378/ches t.14-2241

[2] Rosenquist RW. Postoperative pain control in the thoracic surgical patient. Seminars in Anesthesia, Perioperative Medicine and Pain. 2002; 21(3): 232-240. https://doi.org/10.1053/sane. 2002. 34185

[3] Richard C, Girard F, Ferraro P, et al. Acute postoperative pain in lung transplant recipients. Ann Thorac Surg. 2004; 77(6): 19515. PMid: 15172243. https://doi.org/10.1016/j.athoracs ur.2003.12.038

[4] Cason M, Naik A, Grimm JC, et al. The efficacy and safety of epidural-based analgesia in a case series of patients undergoing lung transplantation. J Cardiothorac Vasc Anesth. 2015; 29(1): 12632. PMid: 25440655. https://doi.org/10.1053/j.jvca. 201 4.07 .023

Published by Sciedu Press
[5] Sabanathan S, Eng J, Mearns AJ. Alterations in respiratory mechanics following thoracotomy. J R Coll Surg Edinb. 1990; 35(3): 144-50.

[6] Katz J, Jackson M, Kavanagh BP, et al. Acute pain after thoracic surgery predicts long-term post-thoracotomy pain. Clin J Pain. 1996; 12(1): 50-5. PMid: 8722735 . https://doi.org/10.1097/0000 2508-199603000-00009

[7] Feltracco P, Barbieri S, Milevoj M, et al. Thoracic epidural analgesia in lung transplantation. Transplant Proc. 2010; 42(4): $1265-$ 9. PMid: 20534277. https://doi.org/10.1016/j.transpro ceed.2010.03.109

[8] Peng Z, Li H, Zhang C, et al. A retrospective study of chronic post-surgical pain following thoracic surgery: prevalence, risk factors, incidence of neuropathic component, and impact on qualify of life. PLoS ONE. 2014; 9(2): e90014. PMid: 24587187. https://doi.org/10.1371/journal.pone.0090014

[9] Ungprasert P, Cheungpasitporn W, Crowson CS, et al. Individual non-steroidal anti-inflammatory drugs and risk of acute kidney injury: A systematic review and meta-analysis of observational stud- 
ies. Eur J Intern Med. 2015; 26(4): 285-91. PMid: 25862494. https://doi.org/10.1016/j.ejim.2015.03.008

[10] Schafer AI. Effects of nonsteroidal antiinflammatory drugs on platelet function and systemic hemostasis. J Clin Pharmacol. 1995; 35(3): 209-19. PMid: 7608308. https ://doi.org/10.1002/j.1552-4 604.1995.tb04050.x

[11] Chen MR, Dragoo JL. The effect of nonsteroidal anti-inflammatory drugs on tissue healing. Knee Surg Sports Traumatol Arthrosc. 2013; 21(3): 540-9. PMid: 22744434. https ://doi.org/10.1007/s0 0167-012-2095-2

[12] Krakowski JC, Arora H. Con: Thoracic epidural block is not superior to paravertebral blocks for open thoracic surgery. J Cardiothorac Vasc Anesth. 2015; 29: 1720-1722. PMid: 26386503. https://doi.org/10.1053/j.jvca.2015.06.012

[13] Teeter EG, Kumar PA. Pro: Thoracic epidural block is superior to paravertebral blocks for open thoracic surgery. J Cardiothorac Vasc Anesth. 2015; 29: 1717-1719. PMid: 26386502. https: //doi.org/10.1053/j.jvca.2015.06.015

[14] Hutchins J, Apostolidou I, Shumway S, et al. Paravertebral Catheter Use for Postoperative Pain Control in Patients After Lung Transplant Surgery: A Prospective Observational Study. J Cardiothorac Vasc Anesth. 2017; 31(1): 142-146. PMid: 27543993. https : //doi.org/10.1053/j.jvca.2016.05.006

[15] Yeung JHY, Gates S, Naidu BV, et al. Paravertebral block versus thoracic epidural for patients undergoing thoracotomy (Review). Cochrane Database Syst Rev 2:CD009121. 2016. https: //doi.org/10.1002/14651858.CD009121.pub2

[16] Wu CL, Jani ND, Perkins FM, et al. Thoracic epidural analgesia versus intravenous patient-controlled analgesia for the treatment of rib fracture pain after motor vehicle crash. J Trauma. 1999; 47: 564.
PMid: 10498316. https://doi.org/10.1097/00005373-199 909000-00025

[17] Sztain JF, Gabriel RA, Said ET. Thoracic Epidurals are Associated With Decreased Opioid Consumption Compared to Surgical Infiltration of Liposomal Bupivacaine Following Video-Assisted Thoracoscopic Surgery for Lobectomy: A Retrospective Cohort Analysis. J Cardiothorac Vasc Anesth. 2018. PMid: 30145075. https://doi.org/10.1053/j.jvca.2018.06.013

[18] Farquhar JM, Smith PJ, Snyder L, et al. Patterns and predictors of pain following lung transplantation. Gen Hosp Psychiatry. 2018; 50: 125-130. PMid: 29190571. https://doi.org/10.1016/j.genh osppsych.2017.11.007

[19] Richard C, Girard F, Ferraro P. Acute postoperative pain in lung transplant recipients. Annals Thoracic Surgery. 2004; 77: 19511955. PMid: 15172243. https://doi.org/10.1016/j.athora csur. 2003.12.038

[20] Sentürk M, Ozcan PE, Talu GK, et al. The effects of three different analgesia techniques on long-term postthoracotomy pain. Anesth Analg. 2002; 94(1): 11-5, table of contents. https ://doi.org/10 .1097/00000539-200201000-00003

[21] Michel-cherqui M, Ley L, Szekely B, et al. Prevalence and characteristics of pain in patients awaiting lung transplantation. J Pain Symptom Manage. 2015; 49(3): 548-54. PMid: 25150816. https : //doi.org/10.1016/j.jpainsymman.2014.07.011

[22] Ho AMH, Chung DC, Joynt GM. Neuraxial blockade and hematoma in cardiac surgery: estimating the rare adverse event that has not (yet) occurred. Chest. 2000; 117: 551. PMid: 10669702. https: //doi.org/10.1378/chest.117.2.551

[23] Anegawa S, Hayashi T, Furukawa Y, et al. Spontaneous epidural hematoma after open heart surgery: case report. No Shinkei Geka. 1999; 27(11): 1023-6 